J. Nonlinear Var. Anal. 3 (2019), No. 1, pp. 107-114

Available online at http://jnva.biemdas.com

https://doi.org/10.23952/jnva.3.2019.1.10

\title{
APPROXIMATING FIXED POINTS OF $\rho$-NONEXPANSIVE MAPPINGS BY RK-ITERATIVE PROCESS IN MODULAR FUNCTION SPACES
}

\author{
SAFEER HUSSAIN KHAN ${ }^{1, *}$, HAFIZ FUKHAR-UD-DIN ${ }^{2}$ \\ ${ }^{1}$ Department of Mathematics, Statistics and Physics, Qatar University, Doha 2713, Qatar \\ ${ }^{2}$ Department of Mathematics and Statistics, King Fahd University of Petroleum and Minerals, Dhahran 31261, Saudi Arabia \\ Dedicated to Professor Wataru Takahashi on the occasion of his 75th birthday
}

\begin{abstract}
In this paper, we use the so-called RK-iterative process to approximate fixed points of nonexpansive mappings in modular function spaces. This process converges faster than its several counterparts. This will create some new results in modular function spaces while generalizing and improving several existing results.
\end{abstract}

Keywords. Fixed point; Nonexpansive mapping; Iterative process; Modular function space.

2010 Mathematics Subject Classification. 47H09, 47H10, 54C60.

\section{INTRODUCTION}

Fixed Point Theory has become a very powerful and important tool in the study of nonlinear phenomena. It is a rich area of research and has applications in numerous areas of research and real-world problems. The metric fixed pint theory in Banach spaces has a close interplay between geometric and topological conditions. Fixed point theory in modular function spaces and that in metric spaces are closely related because former provides the modular equivalence of norm and metric concepts. Modular spaces are generalizations of the classical Lebesgue and Orlicz spaces, and mostly the conditions in the setting of modular spaces are more natural and more easily verified than their metric counterparts. We refer the reader to Khamsi and Kozlowski [4] for details.

A vigorous research activity in the area of numerical reckoning of fixed points for suitable classes of nonlinear operators is underway these years; see, for example [11], and for applications to image recovery and variational inequalities, see, for example, [3] and references therein. Existence of fixed points in modular function spaces has been studied by many researchers, see, for example, Khamsi and Kozlowski [4] and the references therein. Dhompongsa et al. [2] proved the existence of fixed point of $\rho$-contractions under certain conditions. Bin Dehaish and Kozlowski [1] approximated fixed points in modular function spaces using Mann and Ishikawa iterative processes. Khan and Abbas [6] initiated work for multivalued mappings in modular function spaces using the Mann iterative process. For some more recent works, we refer the reader to Okeke et al. [7] and Zegeye et al. [12]. Picard, Mann and Ishikawa iterative processes are classical iterative processes. Khan [5] introduced a new iterative process

${ }^{*}$ Corresponding author.

E-mail addresses: safeer@qu.edu.qa (S.H. Khan), hfdin@kfupm.edu.sa (H. Fukhar-ud-din).

Received November 8, 2018; Accepted February 4, 2019.

(C)2019 Journal of Nonlinear and Variational Analysis 
which was a hybrid of Picard and Mann iterative processes. It can be used for approximation of fixed points of certain nonlinear mappings in different ambient spaces. This process is faster and independent of both Mann and Ishikawa iterative processes in the sense that neither reduces to the other under the given conditions. A lot of work since then has been done on various generalizations of this process. For this, we refer the reader to [8] and the references cited therein. Recently, some "modern iterative processes", which make use of "function value" for the whole step at every step along with a part of it, have been considered by several authors. One such process is called the RK-iterative process considered by Ritika and Khan [8] as follows:

$$
\left\{\begin{array}{c}
x_{n+1}=T v_{n}, \\
v_{n}=T\left(\left(1-\alpha_{n}\right) y_{n}+\alpha_{n} T y_{n}\right), \\
y_{n}=T\left(\left(1-\beta_{n}\right) z_{n}+\beta_{n} T z_{n}\right), \\
z_{n}=T\left(\left(1-\gamma_{n}\right) x_{n}+\gamma_{n} T x_{n}\right), n \in \mathbb{N}
\end{array}\right.
$$

This process converges faster for certain mappings; see Ritika and Khan [8] and references cited therein.

In this paper, we use the RK-iterative process to approximate fixed points of $\rho$-nonexpansive mappings in modular function spaces. This will create some new results in modular function spaces while generalizing and improving several existing results.

\section{PRELIMINARIES}

Here is a brief note on modular function spaces to make the discussion self-contained. This has mainly been extracted from Khamsi and Kozlowski [4].

Let $\Omega$ be a nonempty set and $\Sigma$ a nontrivial $\sigma$-algebra of subsets of $\Omega$. Let $\mathscr{P}$ be a $\delta$-ring of subsets of $\Omega$ such that $E \cap A \in \mathscr{P}$ for any $E \in \mathscr{P}$ and $A \in \Sigma$. Let us assume that there exists an increasing sequence of sets $K_{n} \in \mathscr{P}$ such that $\Omega=\cup K_{n}$ (for instance, $\mathscr{P}$ can be the class of sets of finite measure in a $\sigma$-finite measure space). By $1_{A}$, we denote the characteristic function of the set $A$ in $\Omega$. By $\mathscr{E}$, we denote the linear space of all simple functions with supports from $\mathscr{P}$. By $\mathscr{M}_{\infty}$, we denote the space of all extended measurable functions, i.e., all functions $f: \Omega \rightarrow[-\infty, \infty]$ such that there exists a sequence $\left\{g_{n}\right\} \subset \mathscr{E}$, $\left|g_{n}\right| \leq|f|$ and $g_{n}(\omega) \rightarrow f(\omega)$ for all $\omega \in \Omega$.

Definition 2.1. Let $\rho: \mathscr{M}_{\infty} \rightarrow[0, \infty]$ be a nontrivial, convex and even function. We say that $\rho$ is a regular convex function pseudomodular if

(1) $\rho(0)=0$;

(2) $\rho$ is monotone, i.e., $|f(\omega)| \leq|g(\omega)|$ for any $\omega \in \Omega$ implies $\rho(f) \leq \rho(g)$, where $f, g \in \mathscr{M}_{\infty}$;

(3) $\rho$ is orthogonally subadditive, i.e., $\rho\left(f 1_{A \cup B}\right) \leq \rho\left(f 1_{A}\right)+\rho\left(f 1_{B}\right)$ for any $A, B \in \Sigma$ such that $A \cap B \neq \phi, f \in \mathscr{M}_{\infty}$

(4) $\rho$ has the Fatou property, i.e., $\left|f_{n}(\omega)\right| \uparrow|f(\omega)|$ for all $\omega \in \Omega$ implies $\rho\left(f_{n}\right) \uparrow \rho(f)$, where $f \in$ $\mathscr{M}_{\infty}$;

(5) $\rho$ is order continuous in $\mathscr{E}$, i.e., $g_{n} \in \mathscr{E}$, and $\left|g_{n}(\omega)\right| \downarrow 0$ implies $\rho\left(g_{n}\right) \downarrow 0$.

A set $A \in \Sigma$ is said to be $\rho$-null if $\rho\left(g 1_{A}\right)=0$ for every $g \in \mathscr{E}$. A property $p(\omega)$ is said to hold $\rho$ almost everywhere ( $\rho$-a.e.) if the set $\{\omega \in \Omega: p(\omega)$ does not hold $\}$ is $\rho$-null. As usual, we identify any pair of measurable sets whose symmetric difference is $\rho$-null as well as any pair of measurable functions 
differing only on a $\rho$-null set. With this in mind, we define

$$
\mathscr{M}(\Omega, \Sigma, \mathscr{P}, \rho)=\left\{f \in \mathscr{M}_{\infty}:|f(\omega)|<\infty \rho \text {-a.e. }\right\},
$$

where $f \in \mathscr{M}(\Omega, \Sigma, \mathscr{P}, \rho)$ is actually an equivalence class of functions equal $\rho$-a.e. rather than an individual function. Where no confusion exists, we will write $\mathscr{M}$ instead of $\mathscr{M}(\Omega, \Sigma, \mathscr{P}, \rho)$.

It is easy to see that $\rho: \mathscr{M} \rightarrow[0, \infty]$ possesses the following properties:

1. $\rho(0)=0$ iff $f=0 \rho$-a.e.

2. $\rho(\alpha f)=\rho(f)$ for every scalar $\alpha$ with $|\alpha|=1$ and $f \in \mathscr{M}$.

3. $\rho(\alpha f+\beta g) \leq \rho(f)+\rho(g)$ if $\alpha+\beta=1, \alpha, \beta \geq 0$ and $f, g \in \mathscr{M}$.

$\rho$ is called a convex modular if, in addition, the following property is satisfied:

$3^{\prime} . \rho(\alpha f+\beta g) \leq \alpha \rho(f)+\beta \rho(g)$ if $\alpha+\beta=1, \alpha, \beta \geq 0$ and $f, g \in \mathscr{M}$.

Definition 2.2. Let $\rho$ be a regular function pseudomodular. We say that $\rho$ is a regular convex function modular if $\rho(f)=0$ implies $f=0 \rho$-a.e.

The class of all nonzero regular convex function modulars defined on $\Omega$ is denoted by $\Re$.

The convex function modular $\rho$ defines the modular function space $L_{\rho}$ as

$$
L_{\rho}=\{f \in \mathscr{M}: \rho(\lambda f) \rightarrow 0 \text { as } \lambda \rightarrow 0\} .
$$

Generally, the modular $\rho$ is not sub-additive and therefore does not behave as a norm or a distance. However, the modular space $L_{\rho}$ can be equipped with an $F$-norm defined by

$$
\|f\|_{\rho}=\inf \left\{\alpha>0: \rho\left(\frac{f}{\alpha}\right) \leq \alpha\right\} .
$$

In case $\rho$ is convex modular,

$$
\|f\|_{\rho}=\inf \left\{\alpha>0: \rho\left(\frac{f}{\alpha}\right) \leq 1\right\}
$$

defines a norm on the modular space $L_{\rho}$, and is called the Luxemburg norm.

Define

$$
L_{\rho}^{0}=\left\{f \in L_{\rho}: \rho(f, .) \text { is order continuous }\right\}
$$

and the linear space

$$
E_{\rho}=\left\{f \in L_{\rho}: \lambda f \in L_{\rho}^{0} \text { for every } \lambda>0\right\} .
$$

Definition 2.3. $\rho \in \Re$ is said to satisfy the $\Delta_{2}$-condition, if $\sup _{n \geq 1} \rho\left(2 f_{n}, D_{k}\right) \rightarrow 0$ as $k \rightarrow \infty$ whenever $\left\{D_{k}\right\}$ decreases to $\phi$ and $\sup _{n \geq 1} \rho\left(f_{n}, D_{k}\right) \rightarrow 0$ as $k \rightarrow \infty$.

If $\rho$ is convex and satisfies the $\Delta_{2}$-condition, then $L_{\rho}=E_{\rho}$. Moreover, $\rho$ satisfies the $\Delta_{2}$-condition if and only if $F$-norm convergence and modular convergence are equivalent.

Definition 2.4. Let $\rho \in \mathfrak{R}$.

(i) Let $r>0, \varepsilon>0$. Define

$$
D_{1}(r, \varepsilon)=\left\{(f, g): f, g \in L_{\rho}, \rho(f) \leq r, \rho(g) \leq r, \rho(f-g) \geq \varepsilon r\right\} .
$$

Let

$$
\delta_{1}(r, \varepsilon)=\inf \left\{1-\frac{1}{r} \rho\left(\frac{f+g}{2}\right):(f, g) \in D_{1}(r, \varepsilon)\right\} \text { if } D_{1}(r, \varepsilon) \neq \phi
$$


and $\delta_{1}(r, \varepsilon)=1$ if $D_{1}(r, \varepsilon)=\phi$. We say that $\rho$ satisfies $(U C 1)$ if for every $r>0, \varepsilon>0, \delta_{1}(r, \varepsilon)>0$. Note, that for every $r>0, D_{1}(r, \varepsilon) \neq \phi$, for $\varepsilon>0$ small enough.

(ii) We say that $\rho$ satisfies the (UUC1) if, for every $s \geq 0, \varepsilon>0$, there exists $\eta_{1}(s, \varepsilon)>0$ depending only upon $s$ and $\varepsilon$ such that $\delta_{1}(r, \varepsilon)>\eta_{1}(s, \varepsilon)>0$ for any $r>s$.

Note that the $(U C 1)$ implies the $(U U C 1)$.

Definition 2.5. Let $\rho \in \Re$. The sequence $\left\{f_{n}\right\} \subset L_{\rho}$ is called:

- $\rho$-convergent to $f \in L_{\rho}$ if $\rho\left(f_{n}-f\right) \rightarrow 0$ as $n \rightarrow \infty$.

- $\rho$-Cauchy, if $\rho\left(f_{n}-f_{m}\right) \rightarrow 0$ as $n$ and $m \rightarrow \infty$.

Note that, the $\rho$-convergence does not imply the $\rho$-Cauchy since $\rho$ does not satisfy the triangle inequality. In fact, one can show that this will happen if and only if $\rho$ satisfies the the $\Delta_{2}$-condition.

Definition 2.6. Let $\rho \in \mathfrak{R}$. A subset $D \subset L_{\rho}$ is called

- $\rho$-closed if the $\rho$-limit of a $\rho$-convergent sequence of $D$ always belongs to $D$.

- $\rho$-a.e. closed if the $\rho$-a.e. limit of a $\rho$-a.e. convergent sequence of $D$ always belongs to $D$.

- $\rho$-compact if every sequence in $D$ has a $\rho$-convergent subsequence in $D$.

- $\rho$-a.e. compact if every sequence in $D$ has a $\rho$-a.e. convergent subsequence in $D$.

- $\rho$-bounded if $\operatorname{diam}_{\rho}(D)=\sup \{\rho(f-g): f, g \in D\}<\infty$.

A sequence $\left\{t_{n}\right\} \subset(0,1)$ is called bounded away from 0 if there exists $a>0$ such that $t_{n} \geq a$ for every $n \in \mathbb{N}$. Similarly, $\left\{t_{n}\right\} \subset(0,1)$ is called bounded away from 1 if there exists $b<1$ such that $t_{n} \leq b$ for every $n \in \mathbb{N}$. The following lemma can be seen as an analogue of a famous lemma due to Schu [9] in Banach spaces.

Lemma 2.1. [4, Lemma 4.2] Let $\rho \in \Re$ satisfy the (UUC1) and let $\left\{t_{k}\right\} \subset(0,1)$ be bounded away from 0 and 1 . If there exists $R>0$ such that

$$
\limsup _{n \rightarrow \infty} \rho\left(f_{n}\right) \leq R, \limsup _{n \rightarrow \infty} \rho\left(g_{n}\right) \leq R,
$$

and

$$
\lim _{n \rightarrow \infty} \rho\left(t_{n} f_{n}+\left(1-t_{n}\right) g_{n}\right)=R
$$

then

$$
\lim _{n \rightarrow \infty} \rho\left(f_{n}-g_{n}\right)=0
$$

A function $f \in L_{\rho}$ is called a fixed point of $T: L_{\rho} \rightarrow L_{\rho}$ if $f=T f$. The set of all fixed points of $T$ is denoted by $F_{\rho}(T)$.

The $\rho$-distance from an $f \in L_{\rho}$ to a set $D \subset L_{\rho}$ is given as follows:

$$
\operatorname{dist}_{\rho}(f, D)=\inf \{\rho(f-h): h \in D\} .
$$

The following definition is a modular space version of the condition $(I)$ of Senter and Dotson [10]. Let $D \subset L_{\rho}$. A mapping $T: D \rightarrow D$ is said to satisfy the condition $(I)$ if there exists a nondecreasing function $\ell:[0, \infty) \rightarrow[0, \infty)$ with $\ell(0)=0, \ell(r)>0$ for all $r \in(0, \infty)$ such that

$$
\rho(f-T f) \geq \ell\left(\operatorname{dist}_{\rho}\left(f, F_{\rho}(T)\right)\right.
$$

for all $f \in D$. 
Definition 2.7. A mapping $T: D \rightarrow D$ is called a $\rho$-nonexpansive mapping if

$$
\rho(T f-T g) \leq \rho(f-g) \text { for all } f, g \in D .
$$

The following general theorem ([4, Theorem 5.7]) confirms the existence fixed points of $\rho$-nonexpansive mappings.

Theorem 2.1. Assume that $\rho \in \Re$ satisfies the (UUC1). Let $D$ be a $\rho$-closed, $\rho$-bounded convex and nonempty subset of $L_{\rho}$. Then any pointwise asymptotically $\rho$-nonexpansive mapping $T: D \rightarrow D$ has a fixed point. Moreover, the set of all fixed points $F(T)$ is $\rho$-closed and convex.

\section{MAIN RESULTS}

Here we first introduce the following counter part of theRK-iterative process (1.1) in the setting of modular function spaces. For a $\rho$-nonexpansive mapping $T: D \rightarrow D$, we define a sequence $\left\{f_{n}\right\}$ by the following iterative process:

$$
\left\{\begin{array}{c}
f_{n+1}=T g_{n}, \\
g_{n}=T\left(\left(1-\delta_{n}\right) h_{n}+\delta_{n} T h_{n}\right), \\
h_{n}=T\left(\left(1-\eta_{n}\right) k_{n}+\eta_{n} T k_{n}\right), \\
k_{n}=T\left(\left(1-\mu_{n}\right) f_{n}+\mu_{n} T f_{n}\right), n \in \mathbb{N},
\end{array}\right.
$$

where $\left\{\delta_{n}\right\},\left\{\eta_{n}\right\}$ and $\left\{\mu_{n}\right\} \in(0,1)$ are bounded away from both 0 and 1 .

Next, using the above iterative process, we study fixed points of $\rho$-nonexpansive mappings in modular function spaces.

Theorem 3.1. Let $\rho \in \Re$ satisfy the (UUC1) and the $\Delta_{2}$-condition. Let $D$ be a nonempty $\rho$-closed, $\rho$ bounded and convex subset of $L_{\rho}$. Let $T: D \rightarrow D$ be a $\rho$-nonexpansive mapping. Let $\left\{f_{n}\right\} \subset D$ be defined in iterative process (3.1). Then $\lim _{n \rightarrow \infty} \rho\left(f_{n}-q\right)$ exists for all $q \in F_{\rho}(T)$, and $\lim _{n \rightarrow \infty} \rho\left(f_{n}-T f_{n}\right)=0$.

Proof. Since $F_{\rho}(T) \neq \varnothing$ by Theorem 2.1, let $q \in F_{\rho}(T)$. To prove that $\lim _{n \rightarrow \infty} \rho\left(f_{n}-q\right)$ exists for all $q \in F_{\rho}(T)$, we consider

$$
\rho\left(f_{n+1}-q\right)=\rho\left(T g_{n}-T q\right) \leq \rho\left(g_{n}-q\right)
$$

that is,

$$
\rho\left(f_{n+1}-q\right) \leq \rho\left(g_{n}-q\right)
$$

Next, we prove

$$
\begin{aligned}
\rho\left(g_{n}-q\right) & \leq \rho\left[T\left(\left(1-\delta_{n}\right) h_{n}+\delta_{n} T h_{n}\right)-T q\right] \\
& \leq \rho\left(\left(1-\delta_{n}\right) h_{n}+\delta_{n} T h_{n}-q\right) \\
& \left.=\left(1-\delta_{n}\right) \rho\left(h_{n}-q\right)+\delta_{n} \rho\left(T h_{n}-q\right)\right] \\
& \leq\left(1-\delta_{n}\right) \rho\left(h_{n}-q\right)+\delta_{n} \rho\left(h_{n}-q\right) \\
& =\rho\left(h_{n}-q\right) .
\end{aligned}
$$

Similarly,

$$
\rho\left(h_{n}-q\right) \leq \rho\left(k_{n}-q\right)
$$

and

$$
\rho\left(k_{n}-q\right) \leq \rho\left(f_{n}-q\right) .
$$


Hence, we conclude from (3.2) that

$$
\rho\left(f_{n+1}-q\right) \leq \rho\left(f_{n}-q\right) .
$$

Thus $\lim _{n \rightarrow \infty} \rho\left(f_{n}-q\right)$ exists for each $q \in F_{\rho}(T)$. Suppose that

$$
\lim _{n \rightarrow \infty} \rho\left(f_{n}-q\right)=m
$$

where $m \geq 0$.

Next, we prove that $\lim _{n \rightarrow \infty} \rho\left(f_{n}-T f_{n}\right)=0$. Note that the above calculations also give the following inequalities:

$$
\begin{gathered}
\rho\left(k_{n}-q\right) \leq \rho\left(f_{n}-q\right), \\
\rho\left(f_{n+1}-q\right) \leq \rho\left(k_{n}-q\right) .
\end{gathered}
$$

Using (3.3), (3.4) and (3.5), we have

$$
m=\lim _{n \rightarrow \infty} \rho\left(f_{n+1}-q\right) \leq \limsup _{n \rightarrow \infty} \rho\left(k_{n}-q\right) \leq \lim _{n \rightarrow \infty} \rho\left(f_{n}-q\right)=m .
$$

This gives

$$
\lim _{n \rightarrow \infty} \rho\left(k_{n}-q\right)=m
$$

We also have

$$
\limsup _{n \rightarrow \infty} \rho\left(T f_{n}-q\right) \leq \lim _{n \rightarrow \infty} \rho\left(f_{n}-q\right)=m
$$

Moreover,

$$
\begin{aligned}
\rho\left(k_{n}-q\right) & =\rho\left[T\left(\left(1-\mu_{n}\right) f_{n}+\mu_{n} T f_{n}\right)-q\right] \\
& \left.\leq \rho\left[\left(1-\mu_{n}\right) f_{n}+\mu_{n} T f_{n}\right)-q\right] \\
& \leq\left(1-\mu_{n}\right) \rho\left(f_{n}-q\right)+\mu_{n} \rho\left(T f_{n}-q\right) \\
& \leq\left(1-\mu_{n}\right)\left(f_{n}-q\right)+\mu_{n}\left(f_{n}-q\right) \\
& \leq\left(f_{n}-q\right),
\end{aligned}
$$

which yields that

$$
\left.\rho\left(k_{n}-q\right) \leq \rho\left[\left(1-\mu_{n}\right) f_{n}+\mu_{n} T f_{n}\right)-q\right] \leq\left(f_{n}-q\right) .
$$

From (3.3) and (3.6), we obtain that

$$
\lim _{n \rightarrow \infty} \rho\left[\left(1-\mu_{n}\right)\left(f_{n}-q\right)+\mu_{n}\left(T f_{n}-q\right)\right]=m .
$$

Using (3.3), (3.7) and Lemma 2.1, we have

$$
\lim _{n \rightarrow \infty} \rho\left(f_{n}-T f_{n}\right)=0 .
$$

This completes the proof.

Using the above result, we now prove our convergence results as follows.

Theorem 3.2. Let $\rho \in \mathfrak{R}$ satisfy (UUC1) and $\Delta_{2}$-condition. Let $D$ be a nonempty $\rho$-compact and convex subset of $L_{\rho}$. Let $T: D \rightarrow D$ be a $\rho$-nonexpansive mapping. Let $\left\{f_{n}\right\}$ be as defined by the iterative process (3.1). Then $\left\{f_{n}\right\} \rho$-converges to a fixed point of $T$. 
Proof. Since $D$ is $\rho$-compact, there exists a subsequence $\left\{f_{n_{k}}\right\}$ of $\left\{f_{n}\right\}$ such that $\lim _{k \rightarrow \infty}\left(f_{n_{k}}-z\right)=0$ for some $z \in D$. Since $T$ is a $\rho$-nonexpansive mapping, using convexity of $\rho$, we have

$$
\begin{aligned}
\rho\left(\frac{z-T z}{3}\right) & =\rho\left(\frac{z-f_{n_{k}}}{3}+\frac{f_{n_{k}}-T f_{n_{k}}}{3}+\frac{T f_{n_{k}}-T z}{3}\right) \\
& \leq \frac{1}{3} \rho\left(z-f_{n_{k}}\right)+\frac{1}{3} \rho\left(f_{n_{k}}-T f_{n_{k}}\right)+\frac{1}{3} \rho\left(T f_{n_{k}}-T z\right) \\
& \leq \rho\left(z-f_{n_{k}}\right)+\rho\left(f_{n_{k}}-T f_{n_{k}}\right)+\rho\left(f_{n_{k}}-z\right) \\
& \leq 2 \rho\left(z-f_{n_{k}}\right)+\rho\left(f_{n_{k}}-T f_{n_{k}}\right) .
\end{aligned}
$$

Applying Theorem 3.1, $\lim _{n \rightarrow \infty} \rho\left(f_{n_{k}}-T f_{n_{k}}\right)=0$, that is, $\rho\left(\frac{z-T z}{3}\right)=0$. Hence $z$ is a fixed point of $T$. This shows that $\left\{f_{n}\right\} \rho$-converges to a fixed point of $T$.

Theorem 3.3. Let $\rho \in \mathfrak{R}$ satisfy the (UUC1) and the $\Delta_{2}$-condition. Let $D$ be a nonempty $\rho$-closed, $\rho$-bounded and convex subset of $L_{\rho}$. Let $T: D \rightarrow D$ be a $\rho$-nonexpansive mapping satisfying condition (I). Let $\left\{f_{n}\right\}$ be defined by the iterative process (3.1). Then $\left\{f_{n}\right\} \rho$-converges to a fixed point of $T$.

Proof. we know from Theorem 3.1 that $\lim _{n \rightarrow \infty} \rho\left(f_{n}-q\right)$ exists for all $q \in F_{\rho}(T)$. Suppose that

$$
\lim _{n \rightarrow \infty} \rho\left(f_{n}-q\right)>0
$$

If not, $\lim _{n \rightarrow \infty} \rho\left(f_{n}-q\right)=0$ and we are already home. Owing to Theorem 3.1,

$$
\rho\left(f_{n+1}-q\right) \leq \rho\left(f_{n}-q\right)
$$

So, we can write

$$
\operatorname{dist}_{\rho}\left(f_{n+1}, F_{\rho}(T)\right) \leq \operatorname{dist}_{\rho}\left(f_{n}, F_{\rho}(T)\right) .
$$

Thus $\lim _{n \rightarrow \infty} \operatorname{dist}_{\rho}\left(f_{n}, F_{\rho}(T)\right)$ exists. Appealing to Condition $(I)$ and Theorem 3.1, we arrive at

$$
\lim _{n \rightarrow \infty} \ell\left(\operatorname{dist}_{\rho}\left(f_{n}, F_{\rho}(T)\right)\right) \leq \lim _{n \rightarrow \infty} \rho\left(f_{n}-T f_{n}\right)=0 .
$$

Since $\ell$ is a nondecreasing function and $\ell(0)=0$, one obtains

$$
\lim _{n \rightarrow \infty} \operatorname{dist}_{\rho}\left(f_{n}, F_{\rho}(T)\right)=0 .
$$

We now prove that $\left\{f_{n}\right\}$ is a $\rho$-Cauchy sequence in $D$. For this, let $\varepsilon>0$. By (3.8), there exists a constant $n_{0}$ such that, for all $n \geq n_{0}$,

$$
\operatorname{dist}_{\rho}\left(f_{n}, F_{\rho}(T)\right)<\frac{\varepsilon}{2} .
$$

Hence there exists a $y \in F_{\rho}(T)$ such that

$$
\rho\left(f_{n_{0}}-y\right)<\varepsilon .
$$

Now, for $m, n \geq n_{0}$,

$$
\begin{aligned}
\rho\left(\frac{f_{n+m}-f_{n}}{2}\right) & \leq \frac{1}{2} \rho\left(f_{n+m}-y\right)+\frac{1}{2} \rho\left(f_{n}-y\right) \\
& \leq \rho\left(f_{n_{0}}-y\right) \\
& <\varepsilon
\end{aligned}
$$


Hence, by the $\Delta_{2}$-condition, $\left\{f_{n}\right\}$ is a $\rho$-Cauchy sequence in a $\rho$-closed subset $D$ of $L_{\rho}$, and it converges in $D$. Let $\lim _{n \rightarrow \infty} f_{n}=q$. By using (3.8), we have

$$
\operatorname{dist}_{\rho}\left(q, F_{\rho}(T)\right)=\lim _{n \rightarrow \infty} \operatorname{dist}_{\rho}\left(f_{n}, F_{\rho}(T)\right)=0 .
$$

By Lemma 2.1, $F_{\rho}(T)$ is closed. therefore $q \in F_{\rho}(T)$. This proves that $\left\{f_{n}\right\} \rho$-converges to a fixed point of $T$.

\section{Acknowledgment}

The second author would like to acknowledge the support provided by the Deanship of Scientific Research (DSR) at King Fahd University of Petroleum \& Minerals (KFUPM), Saudi Arabia.

\section{REFERENCES}

[1] B.A. Bin Dehaish, W.M. Kozlowski, Fixed point iteration processes for asymptotic pointwise nonexpansive mappings in modular function spaces, Fixed Point Theory Appl. 2012 (2012), Article ID 118.

[2] S. Dhompongsa, T.D. Benavides, A. Kaewcharoen, B. Panyanak, Fixed point theorems for multivalued mappings in modular function spaces, Sci. Math. Jpn. 63 (2006), 161-169.

[3] E.S. Helou, G.T. Herman, C. Lin, M.V.W. Zibetti, Superiorization of preconditioned conjugate gradient algorithms for tomographic image reconstruction, Appl. Anal. Optim. 2 (2018), 271-284.

[4] M.A. Khamsi, W.M. Kozlowski, Fixed Point Theory in Modular Function Spaces, Birkhauser Basel, (2015).

[5] S.H. Khan, A Picard-Mann hybrid iterative process, Fixed Point Theory Appl. 2013 (2013), Article ID 69.

[6] S.H. Khan, M. Abbas, Approximating fixed points of multivalued $\rho$-nonexpansive mappings in modular function spaces, Fixed Point Theory Appl. 2014 (2014), Article ID 34.

[7] G.A. Okeke, S.A. Bishop, S.H. Khan, Iterative approximation of fixed point of multivalued-quasi-nonexpansive mappings in modular function spaces with applications, J. Funct. Spaces 2018 (2018), Article ID 1785702.

[8] Ritika, S.H. Khan. Convergence of RK-iterative process for generalized nonexpansive mappings in CAT(0) spaces, AsianEuropean J. Math. 12 (2019), Article ID 1950077.

[9] J. Schu, Weak and strong convergence to fixed points of asymptotically nonexpansive mappings, Bull. Aust. Math. Soc. 43 (1991), 153-159.

[10] H.F. Senter, W.G. Dotson, Approximating fixed points of nonexpansive mappings, Proc. Am. Math. Soc., 44 (1974), 375-380.

[11] B.S. Thakur, D. Thakur, M. Postolache, A new iterative scheme for numerical reckoning fixed points of Suzuki's generalized nonexpansive mappings, Appl. Math. Comput. 275 (2016), 147-155.

[12] H. Zegeye, W.W. Kassu, M.G. Sangago, Common fixed points of a finite family of multi-valued $\rho$-nonexpansive mappings in modular function spaces, Commun. Optim. Theory 2016 (2016), Article ID 15. 